\title{
Mamdani Fuzzy Model for Learning Activities Evaluation
}

\author{
Isiaka Rafiu M. \\ Dept. Computer, Library and \\ Information Science \\ Kwara State University, Malete
}

\author{
Omidiora Elijah O. \\ Department of Computer \\ Science and Engineering \\ Ladoke Akintola University of \\ Technology, Ogbomoso
}

\author{
Olabiyisi Stephen O. \\ Department of Computer \\ Science and Engineering \\ Ladoke Akintola University of \\ Technology, Ogbomoso
}

\author{
Okediran Oladotun $\mathrm{O}$. \\ Department of Computer \\ Science and Engineering \\ Ladoke Akintola University of \\ Technology, Ogbomoso
}

\begin{abstract}
The intent of this paper is to determine the extent to which fuzzy model could suitably modelled learner activities in Elearning system. However, the paucity of public dataset that meet the exact requirement of this work poses challenges, which necessitate dataset simulation. The detail approach used for the dataset simulation and the fuzzy model were discussed. Construction of the Inference Mechanism using the Relational Calculus and Mamdani approaches were demonstrated. The performance of the simulated model in MATLAB was measured using classifier uncertainty and confusion based metrics. The Mean Absolute Error (MAE) is 10.45; Root Mean Square Error (RMSE) is 8.71. The result shows that Fuzzy logic (White-Box Model) has a low classification error and invariably a higher accuracy for estimating learner activities. Subsequently, the result obtained shall be revalidated using live data of students' activities in an online course. Furthermore, the current Mamdani's model performance shall be compared with its equivalent Neuro_Fuzzy Model. The more efficient of the two models shall be the choice for integration into an Open Source Learning Management System for automatic learning activities evaluation.
\end{abstract}

\section{General Terms}

Student Modelling, Soft Computing.

\section{Keywords}

Learner Activities, Simulated Dataset, Relational Calculus and Fuzzy Logic.

\section{INTRODUCTION}

Learner's attitude and behaviour to studies are valuable pointers to the expected cognitive performance of the students in an educational system. Conventionally, teachers directly observe learners attitude to study and keep monitored activities information in school records. Some of the parameters used for the attitude and behaviour in this context include percentages of class attendance, regularity of assignment submissions and quality of participation in class activities among others. These observed behaviours are great contributors to students test and evaluation [12]. The challenges with the conventional approaches in the area of data gathering, objective data analysis and application limit its adoption and usefulness. However, the advancement in Information and Communication Technology (ICT), and especially the popularity of electronic based learning platforms, provides the required capabilities that can be leveraged to elicit these important metrics for academic support and administration. Presently, most learning management systems (LMS) have inbuilt tools for tracking learners activities into log-files. Unfortunately, the learner activities $\log$ s are usually bewildered collections of massive data which would normally require further distillation and manipulation for a purposeful use.

Consequently, several analytic tools are provided in most of these LMS to elicit and communicate information to the users either statistically or visually from the log data. Any interested user would therefore be required to explore the analytic tools interface, peruse the information and take necessary decisions. However, for obvious reasons, modelling the learner activities or behaviour evaluation process for automatic decisions is highly desirable. A suitable technique for the scenario must be robust with low complexity; great capabilities to accommodate uncertainty and vagueness without loosen its credibility. A fuzzy logic model which is a soft computing technique in the field of Artificial Intelligence (AI) is considered in this study for the modelling of learner activities evaluation. The choice is informed because it is a 'white-box approach' whose source of structural and parameter identification information could be an expert.

Unfortunately, however, it was discovered that public dataset that satisfy the four variables (Reading Time, Assessment Score, Participation Index and Completion Rating) being considered in this work are not readily available. To this end, the required dataset were simulated. Generally, simulated dataset are commonly used in educational related research, just as it is in medicals to facilitate evaluation of models and systems in the earlier stage of development. This is deducible from the third roles for simulated learners in learning stated in [7]. Application of simulated dataset is also demonstrated in [11] [6]. Furthermore, simulated dataset are sometimes adopted with the intent of preventing any perceived abuse of human rights that may be as associated with exposing personal information to the public.

The intent of this paper is to demonstrate the suitability of a fuzzy model for learning activities evaluation. Description of the approach used for the simulation of the required dataset is explained in section II, section III describes fuzzy modeling techniques and section IV has the detailed explanation of the learning activities modelling. Furthermore, section V describes the model simulation in MATLAB. Section VI 
explains the model evaluation metrics. Finally, section VII discusses the findings and gave insight into the directives of future works.

\section{LEARNING ACTIVITIES DATASET SIMULATION}

Data simulation for research purpose is necessitated either by inadequate volume of dataset or absolute absent of suitable dataset for the domain being modelled. The intent in the former case might be for instance, to blow up the size of the dataset for effective operations of a particular classifier. In such a case, some statistical parameters drawn from the available data would be required. So, in line with the concept of "reverse" statistics [5], required volume of simulated dataset can be generated where the statistical value such as mean, variance, standard deviation etc are known. In Statistical Package for Social Sciences (SPSS) a variety (over thirty) of random variates for specified distribution are available. Examples of such functions are NORMAL(stddev), UNIFORM(max, min), RV.POISSON(mean) or RV.LAPLACE(mean,scale). The choice depends on the type of distribution required. Similarly, the four fundamental random number functions for creating array of random numbers in MATLAB are $\operatorname{rand}(), \operatorname{randi}(), \operatorname{randn}()$ and randperm().

The required dataset simulation used in this work is of the second type. The dataset are formulated based on the three step process suggested in [9]. The three processes recommended are selection of a structure to underlay the data, data generation and data formatting. The structure that underlines the dataset in this work was based on the membership function for the four input variables and output variable. See figure 4,5 and 6 . If the representation for completion status $\{0-45,45.1-100\}=\{a, b\}$, the reading time $\{0-30,30.1-70,70.1100\}=\{c, d, e\}$, the participation index time $\{0-30,30.1-70,70.1100\}=\{\mathrm{f}, \mathrm{g}, \mathrm{h}\}$, and the Assessment $\{0-45,45.1-100\}=\{i, j\}$. The total number of possible combination is thirty six (36), which is as follows.

Table 1: Variable Mappings

\begin{tabular}{|l|c|c|c|c|c|c|c|c|}
\hline acfi & acgi & achi & adfi & adgi & adhi & aefi & aegi & aehi \\
\hline acfj & acgj & achj & adfj & adgj & adhj & aefj & aegj & aehj \\
\hline bcfi & bcgi & bchi & bdfi & bdgi & bdhi & befi & begi & behi \\
\hline bcfj & bcgj & bchj & bdfj & bdgj & bdhj & befj & begj & behj \\
\hline
\end{tabular}

Though any of the aforementioned tools could be used for data simulation however in [10], the data table in MS Excel was identified as a tool for executing substantial simulation, without requiring cumbersome processing "tricks" VBA coding etc. Hence, the random number generator in the Microsoft Excel data analysis tools was used.

Accordingly, the required dataset simulation used in this work is of the second type. The dataset were formulated firstly by selecting the membership function for the four input variables and output variable as the structure that underlines the dataset. Secondly, ten thousand $(10,000)$ unique simulated dataset were formulated. The figure is ten folds of one thousand $(1,000)$ which is the number of unique values between 0.1 and 100 at interval of 0.1 . From the 10,000 by 4 matrix dataset formulated the top ten percent (1000 records) was selected for the model evaluation. The selected $10 \%$ was further subdivided to ratio 70:30. The first $70 \%$ (700) was designated as Educational dataset, the remaining 30\% (300) as testing dataset.

The formulated dataset is analogous of the Transformed / Aggregated Activity Log in the Control layer of the Actuator Indicator Model [8]. The composition of simulated dataset is as shown in figure 1 .

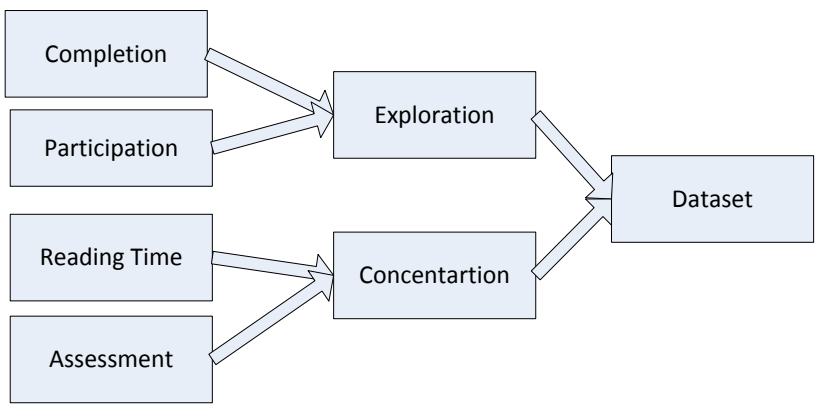

Fig. 1: Composition of the Simulated Dataset

The formulated dataset provides bases for development, validation and testing of White Box implemented as Fuzzy Inference System (FIS). The design details are as follows.

\section{FUZZY MODELLING APPROACH}

Generally mathematical models of real system can be used for simulations, analysis of the system behaviours and design of new processes etc. Modeling is said to be "White box" when it is seen as a conjunction of a thorough understanding of the system's nature, system behaviour and of a suitable mathematical treatment [2]. Fuzzy logic model is a "White box" modelling, it is soft computing approach to modelling linear and non-linear systems. It is a computing technique that is based on the Lofti Zadeh's work in the $60 \mathrm{~s}$. It a paradigm shift from the conventional crisp set to fuzzy set. Fuzzy set is a generalization of the crisp set to a continuous varying degree ranging from 0 to 1 . Fuzzy set are formulated to capture the uncertainty and vagueness in human measurement, decision and actions in any world of discourse. Typically, a fuzzy set A for each of the variables in a universe of discourse $X$, whose membership degree for $\mathrm{x}$ is written as

$A=\{x, \mu A(x) \mid x \in X\}$

A static or a dynamic system which makes use of fuzzy set, fuzzy logic and the corresponding mathematical framework is known as fuzzy system. A fuzzy set can be involved in a fuzzy system, either in the description of the system, in the specification of the system or in the specification of the input, output states variables. The specific importance of fuzzy modeling is more in the domain of incomplete or vague knowledge about the system. The most common fuzzy systems are defined by means of If-then rules, they are said to be a fuzzy rule-based system or simply a fuzzy model [2]. Figure 2 illustrates fuzzy logic control system. 


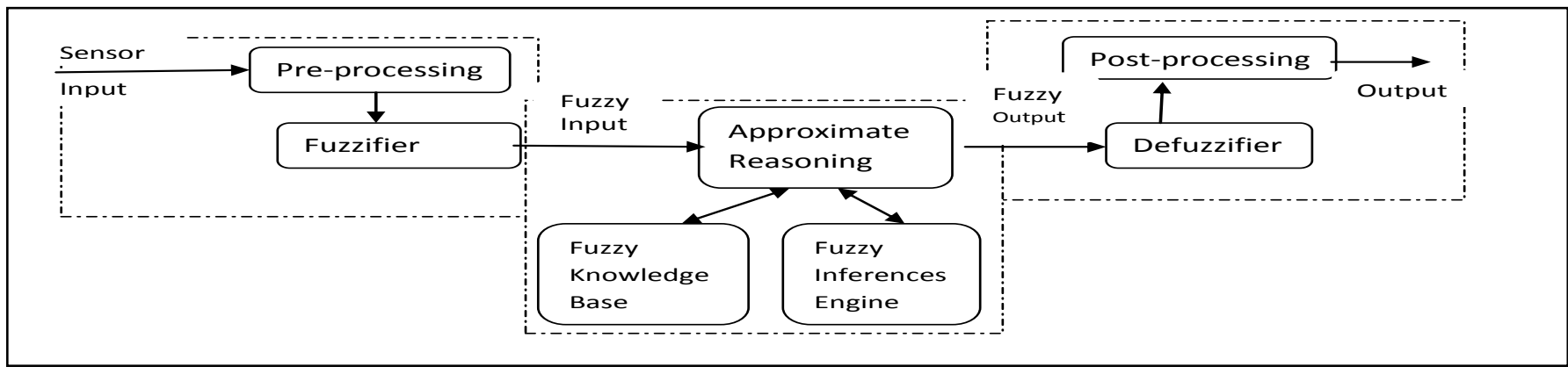

Fig.2: Components of Fuzzy Knowledge Based System [4]

The diagram above illustrates fuzzification, approximate reasoning and defuzzification as the three major parts of a fuzzy logic control system, it is the extended version of the fuzzification, Approximate Reasoning and Defuzzification stages in Fuzzy rule-based system.

In a typical rule based fuzzy systems, the relationships between the variables are represented by means of fuzzy Ifthen rules of the form: If antecedent proposition Then consequent proposition such that if $\bar{x}$ is $A$ then $\bar{y}$ is $B$. $\bar{x}$ and $\bar{y}$ are the input and output linguistic variables while $A$ and $B$ are the input and output linguistic terms respectively. The truth values of the proposition and consequent depends on the degree of match between $\bar{x}$ and $A, \bar{y}$ and $B$ respectively. In fuzzy model, the antecedent proposition is always a fuzzy proposition though the consequent could be either fuzzy proposition or crisp function. When the formal holds the model is said to be a Mamdani (linguistic) fuzzy model, else, it is of the type Takagi-Sugeno fuzzy model.

In the Mamdani Model which is also referred to as linguistic fuzzy model, the system specification is captured in series of if-then rules of the form

$$
R_{i}=\text { if } \bar{x} \text { is } A_{i} \text { then } \bar{y} \text { is } B_{i}
$$

where $\mathrm{i}=1,2, \ldots, \mathrm{k} . \bar{x}, A_{i}, \bar{y}, B_{i}$ are the values of the linguistic variables / linguistic terms for the antecedent and consequents respectively. They are defined in the domains of the respective consequent base variables $x \in X C R^{p}$ (proposition) and $y \in Y C R^{q} \quad$ (consequent). The membership functions of $x$ and $y$ are the mappings $\mu(x): X \rightarrow[0,1]$, and $\mu(y): Y \rightarrow[0,1]$. Similarly, if the domain of the terms are $\mathrm{A}$ and $\mathrm{B}$ then $A_{i} \in A$ and $B_{i} \in$ $B$. The rule base which constitute the qualitative relationship between the model input and output can be represented as

$R=\left\{R_{i} \mid i=1,2,3, \ldots, k\right\}$

For the model to be effective, its inference mechanism must be built on specific Relational representation. The Relational representation of Linguistic model can be computed either by using fuzzy conjunctions $A^{\wedge} B$ or by using fuzzy implications $A \rightarrow B$. Such that each rule in (2) can be regarded as a fuzzy relation $R_{i}:(X \times Y) \rightarrow[0,1]$
Using Mamdani (conjunction) method, the relation $\mathrm{R}$ is computed by the minimum $(\stackrel{)}{)}$ operator:

$R_{i}=A_{i} \times B_{i}$, that is,

$\mu R_{i}(x, y)=\mu A_{i}(x)^{\wedge} \mu B_{i}(y)$

The fuzzy relation $\mathrm{R}$ representing the entire model (2) is given by the disjunction (Union) of the $\mathrm{K}$ individual rule's relations $R_{i}$

$R=\bigcup_{i=1}^{k} R_{i}$, that is $\mu_{R}(x, y)=$ $\frac{\max }{1 \leq i \leq k}\left[\mu A_{i}(x)^{\wedge} \mu B_{i}(y)\right]$

With the entire rule base encoded in the fuzzy relation $\mathrm{R}$, the output of the linguistic model can be computed by the relational max-min composition (o).

$\bar{y}=\bar{x} o R$

However, the domain discretization and storing of relation $\mathrm{R}$ as explained above can be avoided by bypassing the relational calculus when the max-min (mamdani) inference is used. The working of the max-min (mamdani) inference according to [2] can be demonstrated for input fuzzy value $\bar{A}=A^{\prime}$, for which the output value $B^{\prime}$ is given by the relational composition:

$\mu B^{\prime}(y)=\frac{\max }{\times}\left[\mu_{A^{\prime}}(x)^{\wedge} \mu_{R}(x, y)\right]$

Substituting $\left.\mu_{R}(x, y)\right]$ from (4)

$\mu B^{\prime}(y)=\frac{\max }{\times}\left\{\mu_{A^{\prime}}(x)^{\wedge} \frac{\max }{1 \leq i \leq k}\left[\mu A_{i}(x)^{\wedge} \mu B_{i}(y)\right]\right\}$

The order can be changed as below to take care of the max and min operations taken from different domain.

$\mu B^{\prime}(y)=\frac{\max }{1 \leq i \leq k}\left\{\frac{\max }{\times}\left[\mu_{A^{\prime}}(x)^{\wedge} \mu A_{i}(x)^{\wedge} \mu B_{i}(y)\right]\right\}$

If the degree of fulfillment of the ith rule's antecedent is denoted by $B_{i}(y)=\frac{\max }{\times}\left[\mu_{A^{\prime}}(x)^{\wedge} \mu A_{i}(x)\right]$ (10)

The output fuzzy set of the linguistic model becomes

$\mu B_{i}(y)=\frac{\max }{1 \leq i \leq k}\left[B_{\boldsymbol{i}}^{\wedge} \mu B_{i}(y)\right] ; \mathrm{y} \in Y$

The algorithm for the max-min or Mamdani inference is as follows [2], 
Compute the degree of fulfillment by:

$B_{i}=\frac{\max }{\times}\left[\mu_{A^{\prime}}(x)^{\wedge} \mu A_{i}(x)\right], 1 \leq i \leq k$

Derive the output fuzzy sets

$B_{i}: \mu B_{i}^{\prime}(y)=\left[B_{i}^{\wedge} \mu B_{i}(y)\right] ; y \in Y, 1 \leq i \leq k$

Aggregate the output fuzzy set

$B_{i}^{\prime}: \mu B^{\prime}(y)=\frac{\max }{1 \leq i \leq k} \mu B_{i}^{\prime}(y), \mathrm{y} \in Y$

\section{LEARNER'S ACTIVITIES} EVALUATION FUZZY MODEL

The three main stages in fuzzy modelling are $t$ Fuzzification, the Inference and the Defuzzification stages.

\subsection{The Fuzzification}

In this initial step, the input and output terms for the four input variables were determined as follows. The Completion = $\{$ Non Adequate, Adequate $\}=\{0-45,45-100\}$, The Reading Time $=\{$ Low, Medium, High $\}=\{0-30,30-70$, $70-100\}$, The Assessment $=\{$ Unsatisfaction, Satisfaction $\}=$ $\{0-45,45-100\}$, The Participation Index $=\{$ Low, Medium, High $\}=\{0-30,30-70,70-100\}$. Similarly, definition of the output variable was defined as Classification $=\{$ Repeat, Proceed $\}=\{0-45,45-100\}$. The range of the base value for all the variable is put at 100 . This is in line with the practice in the educational sector which is the domain for the model. Gaussian membership function was used for each of the variables. It is given as The Gaussian MF =

$\mu_{A_{i j}}\left(x_{j} ; C_{i j}, \sigma_{i j}\right)=e^{\left(\frac{-\left(x_{j}-c_{i j}\right)^{2}}{2 \sigma_{i j}^{2}}\right)}$

The choice of Gaussian MF is informed by its amenability to adjustment of its parameters $C$ (centre) and $\sigma$ (width) through training.

\subsection{The Inferences}

The thirty six rules based on all the possible combinations of the terms for the four variables are formulated. Excerpt of expert's formulated Fuzzy rules are:

i. If (Completion is NonAdequate) and (ReadingTime is Low) and (ParticipationIndex is Low) and (Assessment is Unsatisfactory) then (Classification is Repeat)

ii. If (Completion is Adequate) and (ReadingTime is Medium) and (ParticipationIndex is Low) and (Assessment is Satisfactory) then (Classification is Proceed)

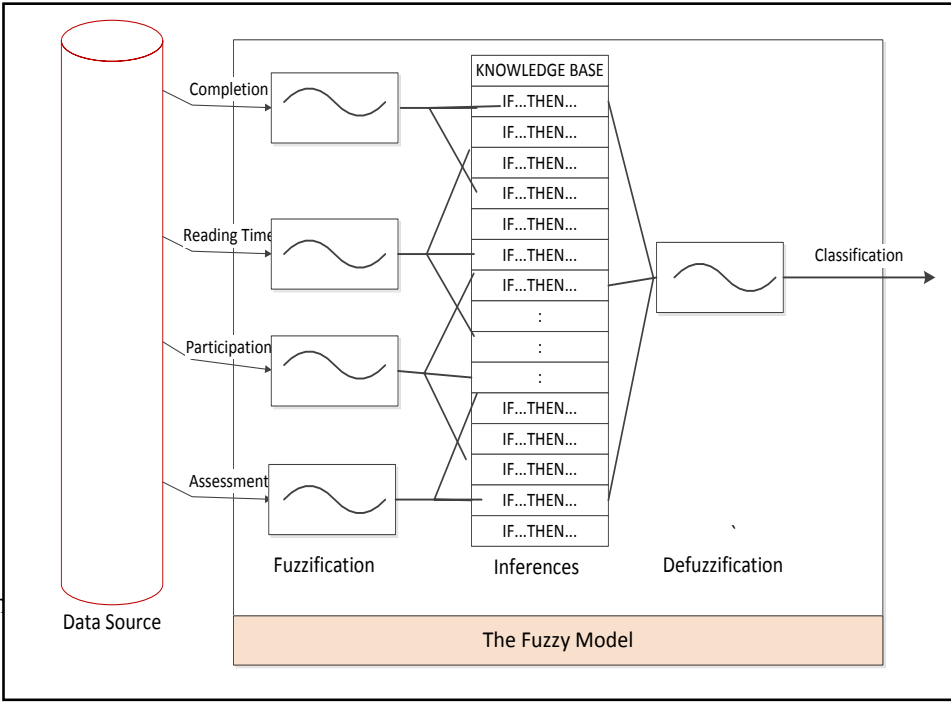

Fig. 3: Fuzzy Model for Learner Activities Evaluation.

The Fuzzy Model for Learners Activities Evaluation is as shown in figure 3 . The membership functions and the discretizations for each of the input and output variable are as follows:

Figure 4 shows the Gaussian Membership Function for course completion variable.

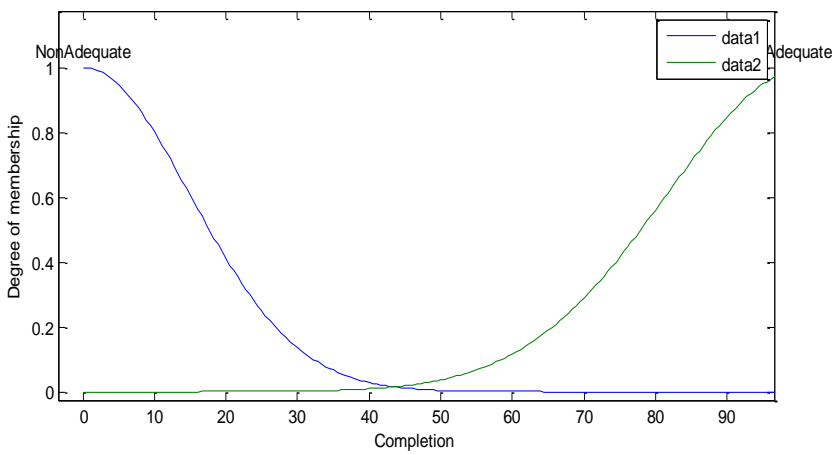

Fig. 4: Completion Membership Function

The discretization for the above membership function is shown in table 2 below.

Table 2: Discretization of Completion Membership Function

\begin{tabular}{|l|l|l|l|l|l|}
\hline C & 0 & 25 & 50 & 75 & 100 \\
\hline NA & 1 & 0.4 & 0 & 0 & 0 \\
\hline A & 0 & 0 & 0.1 & 0.3 & 1 \\
\hline Key: C : Completion variable \\
A : Adequate
\end{tabular}

The membership function for the Assessment variable is similar to that of the completion variable shown above. However, its discretization is as shown in table 3 , below. 
Table 3: Discretization of Assessment Membership Function

\begin{tabular}{|l|l|l|l|l|l|}
\hline$A$ & 0 & 25 & 50 & 75 & 100 \\
\hline$U$ & 1 & 0.4 & 0 & 0 & 0 \\
\hline$S$ & 0 & 0 & 0.1 & 0.3 & 1 \\
\hline
\end{tabular}

Key: A: Assessment variable terms:

U: Unsatisfactory S: Satisfactory

Next is the three terms Gaussian Membership Function for Reading Time variable.

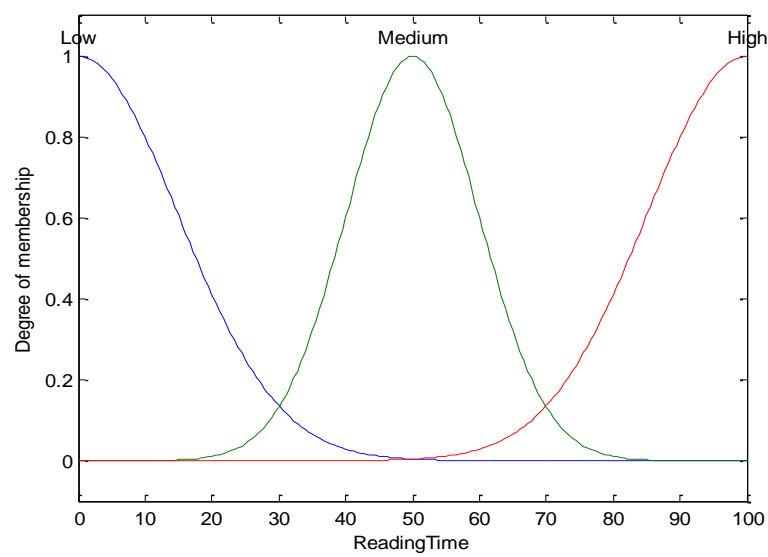

Fig. 5: Reading Time Membership Function and Discretization

The discritization for the Reading Time Membership function shown above is as shown in table 4 below

Table 4: Discretization of Reading Time Membership Function

\begin{tabular}{|l|l|l|l|l|l|}
\hline RT & 0 & 25 & 50 & 75 & 100 \\
\hline L & 1 & 0.2 & 0 & 0 & 0 \\
\hline M & 0 & 0.2 & 1 & 0.2 & 0 \\
\hline H & 0 & 0 & 0 & 0.2 & 1 \\
\hline
\end{tabular}

Key: R: Reading Time variable
L: Low
M: Medium
H: High

The Membership Function and the discritization of the Participation Index is similar to that of the Reading Time variable.

Finally is the Output Membership Functions which is shown in figure 6 below.

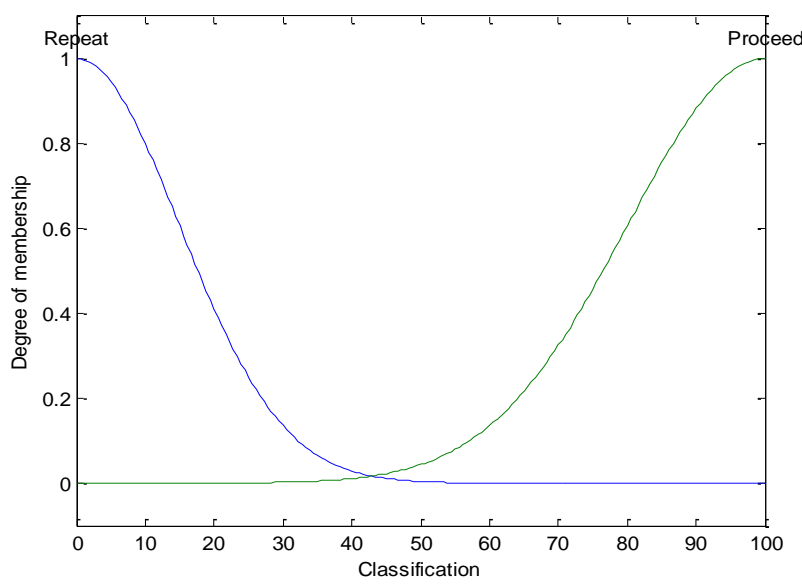

Fig. 6: Classification Membership Function

The fuzzification for the classification Membership function is as shown in table 5 below

Table 5: Discretization for Classification Membership Function

\begin{tabular}{|l|l|l|l|l|c|}
\hline$C L$ & 0 & 25 & 50 & 75 & 100 \\
\hline$R$ & 1 & 0.4 & 0 & 0 & 0 \\
\hline$P$ & 0 & 0 & 0.1 & 0.3 & 1 \\
\hline
\end{tabular}

Key: CL: Classification variable terms:
R: Repeat
P: Proceed

The workings of the linguistic system hinge on the inference algorithm or the inference mechanism which can be constructed using Relational calculus or the Mamdani techniques. The latter minimizes amount of constructions and storage required for the relations.

Using the Relational Calculus approach, the relations for each of the rule $R_{i}, \mathrm{i}=36$ in this case needs to be constructed. Hence, using (4) above,

$$
\begin{aligned}
& R_{i}=C_{i} \times A_{i} \times R T_{i} \times P_{i} \times y= \\
& \mu C_{i} \wedge \mu A_{i}^{\wedge} \mu R_{i} \wedge \mu P_{i}^{\wedge} \mu y \\
& 1 \leq i \leq 36 \text { and } \mathrm{j}=1,2
\end{aligned}
$$

From the discretization of the membership functions and the rules, it is clear that the model is of the Many Input Many Output (MIMO) type. The relation for each rule will be a Cartesian product of all the relevant tuples. The formulation

$$
\begin{array}{ccc}
\text { of } & R_{1} \text { for } \quad \text { instance } & \text { will } \\
& R_{1}=C_{N A} \times A_{u} \times R_{l} \times P_{l} \times y=
\end{array}
$$

$\mu C_{N A}^{\wedge} \mu A_{u}^{\wedge} \mu R_{l}^{\wedge} \mu P_{l}^{\wedge} \mu y$

The discretization for

$\mathrm{R}_{1}=\mu C_{N A^{\wedge}} \mu A_{u}^{\wedge} \mu R_{l}^{\wedge} \mu P_{l}^{\wedge} \mu y$ is as shown in table 6 
Table 6: Discretization for $\mathrm{R}_{1}=\mu \mathrm{C}_{\mathrm{NA}} \wedge \mu \mathrm{A}_{\mathrm{U}} \wedge \mu \mathrm{R}_{\mathrm{L}} \wedge \mu \mathrm{P}_{\mathrm{L}} \wedge \mu \mathrm{Y}$

\begin{tabular}{|l|l|l|l|l|}
\hline 0 & 25 & 50 & 75 & 100 \\
\hline 1 & 0.2 & 0 & 0 & 0 \\
\hline 0.2 & 0.2 & 0 & 0 & 0 \\
\hline 0 & 0 & 0 & 0 & 0 \\
\hline 0 & 0 & 0 & 0 & 0 \\
\hline
\end{tabular}

So using the same approach the relation for other rules is constructed. The fuzzy relation $\mathrm{R}$ representing the entire model is given by the disjunction (Union) of the $\mathrm{K}$ individual rule's relations $R_{i}$ as in (5)

$$
R=\bigcup_{i=1}^{36} R_{i} \text {, that is } \mu_{R}(x, y)=
$$

$\frac{\max }{1 \leq i \leq 36}\left[\mu C_{i}^{\wedge} \mu A_{i}^{\wedge} \mu R_{i}^{\wedge} \mu P_{i}^{\wedge} \mu y\right]$

However, the following is the Mamdani algorithm approach for an input $\bar{x}=A^{\prime}$.

If $B_{i}$ is the degree of fulfilment for each of $R_{i}$.

The degree of fulfilment computation is:

$B_{i}=\frac{\max }{\times}\left[\mu_{A^{\prime}}(x)^{\wedge} \mu C_{i}^{\wedge} \mu A_{i}^{\wedge} \mu R_{i}^{\wedge} \mu P_{i}^{\wedge} \mu y\right]$,

$1 \leq i \leq k$

The output fuzzy sets is derived by

$$
B_{i}: \mu B_{i}^{\prime}(y)=\left[B_{i}^{\wedge} \mu B_{i}(y)\right] ; y \in Y, 1 \leq i \leq k
$$

Aggregate the output fuzzy set

$$
B_{i}^{\prime}: \mu B^{\prime}(y)=\frac{\max }{1 \leq i \leq k} \mu B_{i}^{\prime}(y), \mathrm{y} \in Y
$$

\subsection{The Defuzzification}

The Defuzzification phase is for transforming the fuzzy output to it representative fuzzy value in line with the logic of the system. This is essential in this work also measuring system performance. Most often used defuzzification methods include Centroid, Bisector, Largest of Maximum (LOM), Mean of Maximum (MOM), and Smallest of Maximum (SOM). Centroid of Area (COA) Defuzzification is used and its formular is given as

${ }^{\mathrm{y}} \mathrm{COA}=\frac{\int_{y} \mu_{B}(y) y d y}{\int_{y} \mu_{B}(y) d y}$

\section{THE SIMULATIONS}

MATLAB Version 7 was used to simulate the Fussy Inference System (FIS)

The FIS Editor was launched from the command prompt by executing the fuzzy command.

The parameters were set accordingly. And Method = min, Or Method $=\max$, implication $=\min$, Aggregation $=\max$, Defuzzification $=$ centroid, FIS Type $=$ Mamdani, FIS Name = E_LearningUsers.
The descriptions of the membership function (MF) for the four inputs were defined as follows: The Completion variable: Type= gaussmf; Linguistic Variable NonAdequate $=0-45$; Linguistic Variable Adequate $=45-100 ;$ Range $=\left[\begin{array}{ll}0 & 100\end{array}\right]$ Display Range $=\left[\begin{array}{ll}0 & 100\end{array}\right]$.

The Reading Time: Type $=$ gaussmf; Linguistic Variable Low $=0-30$; Linguistic Variable Medium 30- 70; Variable High $70-100$, Range $=\left[0\right.$ 100]; Display Range $=\left[\begin{array}{l}0 \\ 100\end{array}\right]$.

The Assessment: Type $=$ gaussmf; Variable Unsatisfaction $=0$ - 45; Variable Satisfaction $=45-100 ;$ Range $=\left[\begin{array}{ll}0 & 100\end{array}\right]$; Display Range $=\left[\begin{array}{ll}0 & 100\end{array}\right]$.

Participation Index: Type $=$ gaussmf; Linguistic Variable Low $=0-30$; Linguistic Variable Medium 30- 70; Linguistic Variable High 70- 100, Range $=[0$ 100]; Display Range $=[0$ 100]. Figure 7 below is the overall view of the E-learning Users Fuzzy Inference System.

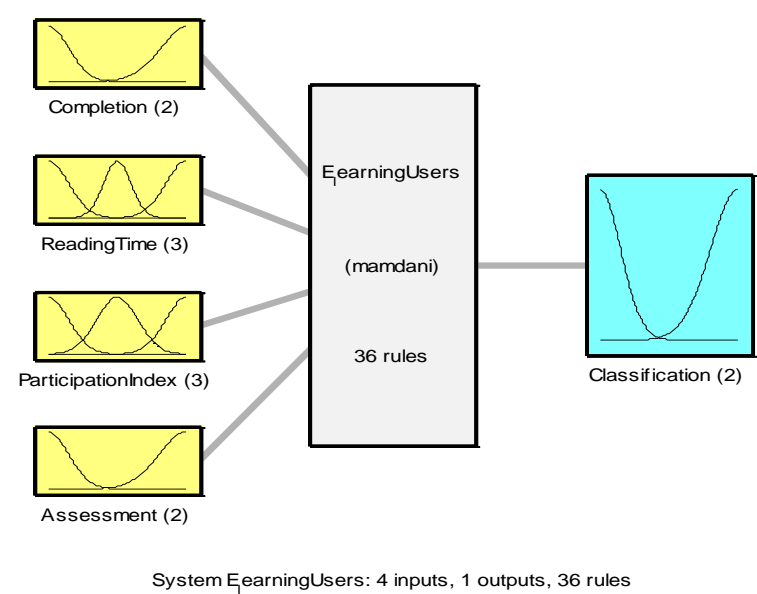

Fig. 7: Formulation of FIS Model

The Rule Editor was used to define the thirty six (36) rules estimated from Decision Tree and outlined in the Rule Table as in [8]. Equal weight of one (1) was assigned to each of the rules.

Figure 8 is the Surface View of the Reading Time, Completion and Classification reflecting the relationship.

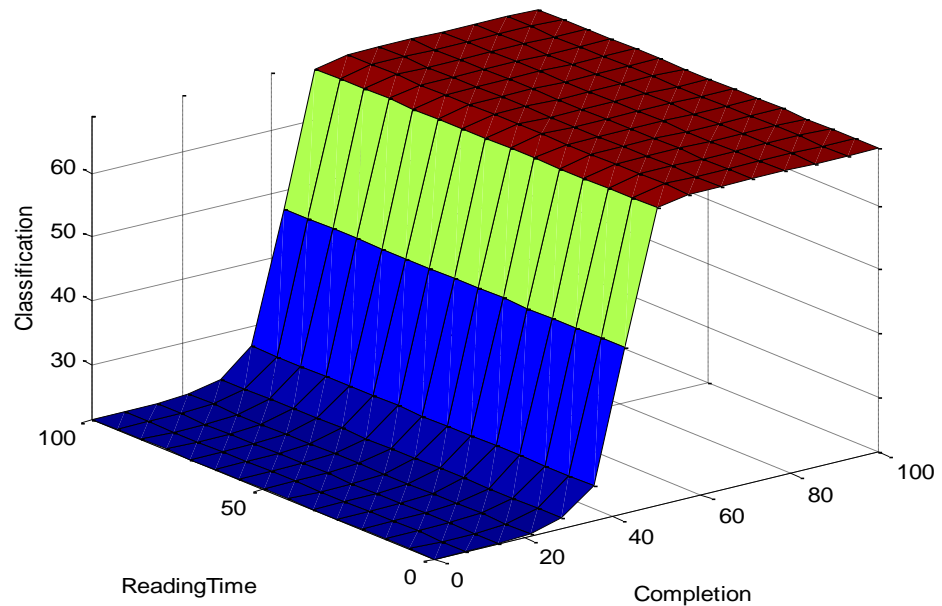

Fig. 8: Surface Viewer for the Reading Time, Completion and Classification variables 
The FIS: e_learningUsers was evaluated using the following readfis and evalfis functions in MATLAB:

\section{> fuzzy e_learningusers \\ > fismat=readfis('e_learningusers')}

fismat $=$

name: 'E_learningUsers'

type: 'mamdani'

$$
\begin{aligned}
& \text { andMethod: 'min' } \\
& \text { orMethod: 'max' } \\
& \text { defuzzMethod: 'centroid' } \\
& \text { impMethod: 'min' } \\
& \text { aggMethod: 'max' }
\end{aligned}
$$

input: [1x4 struct]

output: [1x1 struct]

rule: [1x36 struct]

Table 7 below shows the output of the system. The first four columns are the input values; the last column is the defuzzified output. Each row is an instance or record of a student.

Table 7: Sample FIS Input and Output

\begin{tabular}{lllll}
\multicolumn{2}{l}{ > evalfis } & \multicolumn{2}{l}{ ans $=$} \\
$([29.27$ & 3 & 63.2 & 48 & 24.3198 \\
27.97 & 33.1 & 73 & 2.9 & 18.0210 \\
41.77 & 18.1 & 73.2 & 72.9 & 42.6708 \\
14.17 & 48 & 2.9 & 2.9 & 13.1286 \\
32.97 & 78.1 & 2.9 & 48 & 26.3635 \\
41.82 & 53.2 & 33 & 78.2 & 42.8774 \\
49.35 & 83.3 & 63.2 & 48 & 66.9824 \\
34.3 & 58.4 & 73 & 2.9 & 23.0425 \\
$:$ & $:$ & $:$ & $:$ ],fismat $)$ & $:$
\end{tabular}

\section{PERFORMANCE EVALUATION CRITERIA}

The performance of the machine learning models was based on least values of Mean Absolute Error (MAE), Root MeanSquared Error (RMSE) and the Accuracy.

The Mean Absolute Error (MAE): is the average of the difference between predicted and the actual value in all test cases [1]. It is the average prediction error. Assuming that the actual output is a, and the expected output is c, the formula for calculating MAE is:

$$
\frac{\left|\mathrm{a}_{1}-\mathrm{c}_{1}\right|+\left|\mathrm{a}_{2}-\mathrm{c}_{2}\right|+\left|\mathrm{a}_{3}-\mathrm{c}_{3}\right|+\cdots+\left|\mathrm{a}_{\mathrm{n}}-\mathrm{c}_{\mathrm{n}}\right|}{\mathrm{n}}
$$

The Root Mean-Squared Error (RMSE): is a frequently used measure of differences between values predicted by a model or estimator and the values actually observed (expected) values (Challagula et, al., 2005). It is the square root of the

\begin{tabular}{|c|c|c|c|c|}
\hline $\begin{array}{l}\text { Expected } \\
\text { Output(c) }\end{array}$ & FIS(a) & $|a-c|$ & $(a-c)^{\wedge} 2$ & \\
\hline 35.87 & 24.32 & 11.55 & 133.41 & \\
\hline 34.25 & 18.02 & 16.23 & 263.38 & \\
\hline 51.5 & 42.67 & 8.83 & 77.95 & \\
\hline 17 & 13.13 & 3.87 & 14.99 & \\
\hline 40.5 & 26.36 & 14.14 & 199.84 & \\
\hline 51.56 & 42.88 & 8.68 & 75.39 & \\
\hline 60.97 & 66.98 & 6.01 & 36.15 & \\
\hline 42.15 & 23.04 & 19.11 & 365.10 & \\
\hline 73.5 & 74.54 & 1.04 & 1.08 & \\
\hline 4.78 & 12.37 & 7.59 & 57.55 & \\
\hline 34.5 & 27.32 & 7.18 & 51.62 & \\
\hline \multirow[t]{3}{*}{ : } & : & : & $:$ & \\
\hline & $\sum(|a-c|)$ & 1568.23 & 19662.50 & $\sum(\mathrm{a}-\mathrm{c})^{\wedge} 2$ \\
\hline & $\sum_{/ \mathrm{n}}(|\mathrm{a}-\mathrm{c}|)$ & $\begin{array}{l}8.71 \\
\text { (MAE) }\end{array}$ & $\begin{array}{l}10.45 \\
\text { (RMSE) }\end{array}$ & $\begin{array}{l}\sqrt{ }\left(\left(\sum(a-c)\right.\right. \\
\wedge 2) / n)\end{array}$ \\
\hline
\end{tabular}

mean square error (Ardil and Sandhu, 2010). The formular is as shown below:

$$
\sqrt{\frac{\left(a_{1}-c_{1}\right)^{2}+\left(a_{2}-c_{2}\right)^{2}+\left(a_{3}-c_{3}\right)^{2}+\cdots+\left(a_{n}-c_{n}\right)^{2}}{n}}
$$

Table 8: Performance Evaluation Calculation

\section{FUTURE WORK}

Moving forward in this work, the current results shall be revalidated using live data of students enrolled in an online course in Moodle Learning Management System. Comparison shall be made between the performance of the current fuzzy logic model and the equivalent Neuro-fuzzy model. Subsequently the more efficient of the two models shall be integrated into the Moodle LMS for automatic Evaluation of Learning Activities.

\section{REFERENCES}

[1] Ardil, E., and Sandhu, P.S. 2010. A soft computing approach for modeling of severity of faults in software systems. International Journal of Physical Sciences. 5(2), 74-85. Retrieved from http://www.academicjournals.org/IJPS.

[2] Babuska, R. 2001. Fuzzy systems, modeling and identification. Technical report, Delft University of Technology. Netherland.

[3] Challagulla, V., Bastani, F., Yen, I., and Paul, R. 2005. Empirical assessment of machine learning based software defect prediction techniques. 10th IEEE International Workshop on Object-Oriented Real-Time Dependable Systems. WORDS 2005. 263-270.

[4] Devendra, K.C. 2010. Modeling and simulation of system using MATLAB and SIMULINK. CRC Press, Taylor \& Francis Group, Boca Raton. 
[5] Garson, D.G. 2010. Creating simulated datasets. Statistics Associates Publishing- Blue Book Series, 274 Glenn Drive, Ashebro, NC 272905 USA. Retrieved from www. statisticsassociations.com

[6] Liang, J. 2010. Data simulation: create a dummy dataset using clinical administrative database. Technical Report, Canadian Institute for Health Information.

[7] McCalla G., and Champaign, J. 2013. AIED 2013 simulated learners workshop. Artificial Intelligence in Education, Lecture Notes In Computer Science. 7926, 954-955

[8] Omidiora, E.O, Olabiyisi, S.O, Okediran, O.O., and Isiaka, R.M. 2013. Learner activities evaluation model: A neuro-fuzzy approach. International Journal of eEducation, e-Business, e-Management and e-Learning. 3(5), 421-424
[9] Open Mx Project. 2013. Open Mx- Advanced structural equation modeling. Retrieved from openmx.psyc.virginia.edu

[10] Switzer, D. 2013. Simulation using Excel: tricks, trial and trends. Retrieved from https://sites,google.com/site/dennissweitzer/home

[11] Vanlehn, K. Ohlsson, S., and Nason, R. 1994. Applications of simulated students: An exploratory. Journal of Artificial Intelligence in Education, 5(2), 135175

[12] Yildiz, O., Bal, O., and Gulsecen, S. 2013. Improved fuzzy modelling to predict the academic performance of distance education students. The International Review of Research in Open and Distance Learning. 14(5), 144-164 